\title{
rs78378222 polymorphism in the 3 '-untranslated region of TP53 contributes to development of age-associated cataracts by modifying microRNA-125b-induced apoptosis of lens epithelial cells
}

\author{
YANG ZHAO, XIAO LI and SIQUAN ZHU \\ Beijing Tongren Eye Center, Beijing Key Laboratory of Ophthalmology and Visual Science, \\ Beijing Tongren Hospital, Capital Medical University, Beijing 100073, P.R. China
}

Received May 29, 2015; Accepted April 29, 2016

DOI: $10.3892 / \mathrm{mmr} .2016 .5465$

\begin{abstract}
MicroRNAs (miRNAs) negatively regulate the expression of the target genes by binding to 'seed sequences' in the 3'-untranslated region (3'-UTR) mRNA transcripts, and the variants within or nearby 'seed sequences' may compromise or enhance miRNA/mRNA interaction leading to either 'loss-of-function' or 'gain-of-function' effects. Cataracts are the leading cause of blindness worldwide and are characterized by progressive aggregation and precipitation of lens proteins, and the development of age-related cataracts is associated with dysregulated cellular activities of lens epithelial cells. Luciferase assays and online miRNA databases were used to validate that tumor protein p53 (TP53) is the target gene of miR-125b. Furthermore, reverse transcription-quantitative polymerase chain reaction and western blotting were conducted to detect expression levels of miR-125b and TP53 in different groups of cells transfected with miR-125b mimics or inhibitors. In addition, flow cytometry analysis and the MTT assay were conducted to detect the effects of miR-125b on apoptosis and cell viability. The current study demonstrated that the rs78378222 polymorphism minor allele introduces a novel potential miR-125b binding site in the TP53 3'-UTR with a consecutive 8-bp perfect match, creating a 'gain-of-function' variant and affecting the regulation of TP53 expression. A luciferase assay demonstrated that transfection of lens epithelial cells with wild type TP53 3'-UTR significantly reduced the luciferase activity of the miR-125b overexpressing cells compared with scramble controls. In addition, the luciferase activity of miR-125b overexpressing cells transfected with the
\end{abstract}

Correspondence to: Dr Siquan Zhu, Beijing Tongren Eye Center, Beijing Key Laboratory of Ophthalmology and Visual Science, Beijing Tongren Hospital, Capital Medical University, 1 Dongjiaominxiang, Beijing 100073, P.R. China

E-mail: siquan_66@163.com

Key words: rs78378222, tumor protein p53, age-associated cataracts, microRNA-125b, apoptosis construct containing the rs78378222 polymorphism minor allele was also reduced compared with cells transfected with the wild type 3'-UTR. Furthermore, it was demonstrated that the expression level of miR-125 was comparable in epithelial cells from patients with age-associated cataracts and controls, whereas the expression level of TP53 was significantly higher in the cataract group compared with controls. Additionally, transfection with $50 \mathrm{nM}$ miR-125b mimics markedly reduced the mRNA and protein expression levels of TP53 in the cultured lens epithelial cells, and miR-125b significantly induced apoptosis in the epithelial cells compared with negative control cells. In conclusion, TP53 was identified as a target of miR-125b, and the minor allele of the rs78378222 polymorphism promoted the miR-125b/TP53 mRNA interaction.

\section{Introduction}

Cataracts are a typical age-correlated condition and the leading cause of visual loss worldwide (1). Previous studies have demonstrated that age-correlated cataracts cause approximately half of all cases of visual loss (2-4). A previous epidemiological study demonstrated that $96 \%$ of people aged $>60$ years have different types or severities of lens opacity (5). According to estimates by the World Health Organization, the number of people with cataract blindness worldwide will reach 40 million by 2025 (6). Age-correlated cataracts cause severe visual impairment, reduce quality of life, and become a burden on social economy and health resources. As an important component of the visual refraction system, human lenses are relatively isolated tissues surrounded by the vitreous body and the aqueous humor. Lenses, which are transparent in younger individuals, change with age. These changes begin with the development of a compact, hard nucleus and regional opacity, which result in the occurrence of pathological cataracts.

MicroRNAs (miRNAs), a class of endogenous small non-coding RNAs of 20-25 nucleotides, modulate the expression of genes at the post-transcriptional level (7). miRNAs bind to complementary sequences present in the 3'-untranslated regions (3'-UTR) of target gene mRNAs and, thus, regulate translational interference or degradation of mRNAs (8). Several miRNAs have previously been detected 
in animal eyes $(9,10)$; miRNA-31 (miRNA-31), miR-184, miR-204, miR-125b, miR-26a, let-7b and others were identified in the lens; miR-26a, miR-24, miR-31 miR-184 and miR-205 were identified in the cornea; and miR-181a, miR-124a, miR-182, miR-183, miR-125b and miR-30 were demonstrated to be produced in the retina $(9,10)$. Accumulating evidence has demonstrated that abnormal expression of miRNAs is closely correlated with the pathogenesis of a wide range of age-associated conditions (11-14), including as cataracts (15). miRNA studies have provided novel insights to the treatment of such diseases $(16,17)$.

Variants in primary miRNAs or in the 3'-UTR of the target gene may interfere with the production of the miRNA or the binding between miRNA and mRNA of the target gene. miRNAs negatively regulate the expression of target genes by binding to the 'seed sequence' in the 3'-UTR of the genes, and the variants within or nearby the seed sequence may inhibit or enhance miRNA/mRNA interaction leading to either 'loss-of-function' or 'gain-of-function'. The present study investigated the potential effect of the rs78378222 polymorphism $(18,19)$ in the 3 '-UTR of tumor protein p53 (TP53) on the miR-125b-induced apoptosis of lens epithelial cells, and the association between the rs78378222 polymorphism and the risk of age-associated cataracts.

\section{Materials and methods}

Study population. Lens epithelial cells were collected as previously described (20) from 12 patients with cataracts and 12 normal controls receiving eye surgery at Beijing Tongren Hospital (Beijing, China). The study protocol followed the guidelines of the Declaration of Helsinki (21) and was approved by the institutional review board of Beijing Tongren Hospital. Following a full explanation of the surgical procedures and possible complications, all patients provided written informed consent. Patients were selected based on clinically observable nuclear cataracts of grade 2 or 3 according to the Lens Opacities Classification System III (22). Exclusion criteria included cataract hardness greater than grade 3. Patients with type 1 diabetes mellitus (DM), rheumatologic disease and other systemic diseases, with the exception of type $2 \mathrm{DM}$, were also excluded. SRA01/04 cells were obtained from the American Type Culture Collection (Manassas, VA, USA) and cultured in RPMI-1640 medium (Thermo Fisher Scientific, Inc., Waltham, MA, USA) containing $100 \mathrm{mg} / \mathrm{ml}$ streptomycin, $100 \mathrm{U} / \mathrm{ml}$ penicillin and $10 \%$ fetal calf serum (Invitrogen; Thermo Fisher Scientific, Inc.) in a humidified atmosphere of $5 \% \mathrm{CO}_{2}$ at $37^{\circ} \mathrm{C}$.

DNA sequencing and cell biology. A DNA extraction kit (Qiagen, Inc., Valencia, CA, USA) was used to extract genomic DNA from patient lens cells. The Sanger method was used to bidirectionally sequence the TP53 3'-UTRs (Beijing Tongren Hospital). A TP53 reference sequence (NM_000546.5; genome.ucsc.edu) was compared with all sequences and used to determine the genotype of the polymorphism.

Reverse transcription-quantitative polymerase chain reaction $(R T-q P C R)$. TRIzol reagent (Invitrogen; Thermo Fisher Scientific, Inc.) was used to extract total RNA from cataractous lens samples and cultured SRA01/04 epithelial cells according to the manufacturer's instructions. A UV-Vis spectrophotometer (UV-1800; Shimadzu Corporation, Kyoto, Japan) was used to determine the quality of RNA. Agarose gel (1.5\%) electrophoresis with 260/280 values between 1.8 and 2.0 was used to confirm RNA integrity. PrimerScript RT reagent kit (Takara Biotechnology Co., Ltd., Dalian, China) was used produce cDNA from 1 ng RNA for RT-qPCR analysis. An ABI 7500 cycler (Applied Biosystems; Thermo Fisher Scientific, Inc.), RT primer and TaqMan probes (Guangzhou RiboBio Co., Ltd., Guangzhou, China) were used to determine the expression of miR-125b and TP53. RNA U6 small nuclear 2 was used as the endogenous reference control. The conditions for the RT reaction were $65^{\circ} \mathrm{C}$ for $5 \mathrm{~min}, 25^{\circ} \mathrm{C}$ for $10 \mathrm{~min}, 42^{\circ} \mathrm{C}$ for $1 \mathrm{~h}$ and $75^{\circ} \mathrm{C}$ for $10 \mathrm{~min}$. The PCR cycling conditions were as follows: $95^{\circ} \mathrm{C}$ for $5 \mathrm{~min} ; 40$ cycles of $95^{\circ} \mathrm{C}$ for $10 \mathrm{sec}$, and $60^{\circ} \mathrm{C}$ for $1 \mathrm{~min}$. RT-qPCR analysis was performed twice on three independent specimens. The primer (Shanghai Jieli Biotechnology Co., Ltd., Shanghai, China) sequences were as follows: Forward, 5'-TCAGTTTGCTGTTCTGGGTG-3' and reverse, 5'-CGGTTGGCTGGAAAGGAG-3' for GAPDH; forward, 5'-CCCCTCTGAGTCAGGAAACA-3' and reverse, 5'-AGA CAGAAGGGCCTGACTCA-3' for TP53; forward, 5'-TCA GTTTGCTGTTCTGGGTG-3' and reverse, 5'-CGGTTGGCT GGAAAGGAG-3' for U6; and forward, 5'-UCCCUGAGA CCCUAACUUGUGA-3' and reverse, 5'-ACAAGUUAGGGU CUCAGGCACU-3' for miR-125b. The equation $R Q=2^{-\Delta \Delta C a}$ was used to calculate the relative abundance of miR-125b and TP53 mRNA in cell lines and tissues (23). GraphPad Prism 5 software (GraphPad Software, Inc., La Jolla, CA, USA) was used to produce graphs and perform analyses.

Luciferase assay. Human genomic DNA was used for amplification of the 3'-UTR of the TP53 gene containing conserved binding sites for miR-125b. Following amplification, the fragments were implanted into the pmiR-RB-REPORT vector (Guangzhou RiboBio Co., Ltd.). A mutant 3'-UTR fragment containing mutations in seed binding sites was obtained using a QuikChange Site-Directed Mutagenesis kit (Agilent Technologies, Inc., Santa Clara, CA, USA), according to the manufacturer's protocols, to introduce deletions in the 3'-UTR of TP53. Similarly, the TP53-3'-UTR mutant fragment was inserted into the same sites of the pmiR-RB-REPORT control vector. For reporter assays, SRA01/04 cells were plated in 24-well plates and cultured overnight in Iscove's modified Dulbecco's medium (Invitrogen; Thermo Fisher Scientific, Inc.). Following incubation, the cells were cotransfected with miR-125b mimic/mimic control and wild-type/mutant reporter plasmid (Guangzhou RiboBio Co., Ltd.) using Lipofectamine 2000 (Invitrogen; Thermo Fisher Scientific, Inc.). At $48 \mathrm{~h}$ post-transfection, the Dual-Luciferase Reporter assay system (Promega Corporation, Madison, WI, USA) was used to determine luciferase activity according to the manufacturer's protocol. The GloMax ${ }^{\mathrm{TM}} 96$ Microplate Luminometer (Promega Corporation) was used to measure luciferase activity. The endogenous control was Renilla luciferase plasmid. Each experiment was conducted with three independent specimens and repeated twice.

Apoptosis analysis. At $48 \mathrm{~h}$ following transfection, an apoptosis assay was performed on SRA01/04 lens epithelial cells 


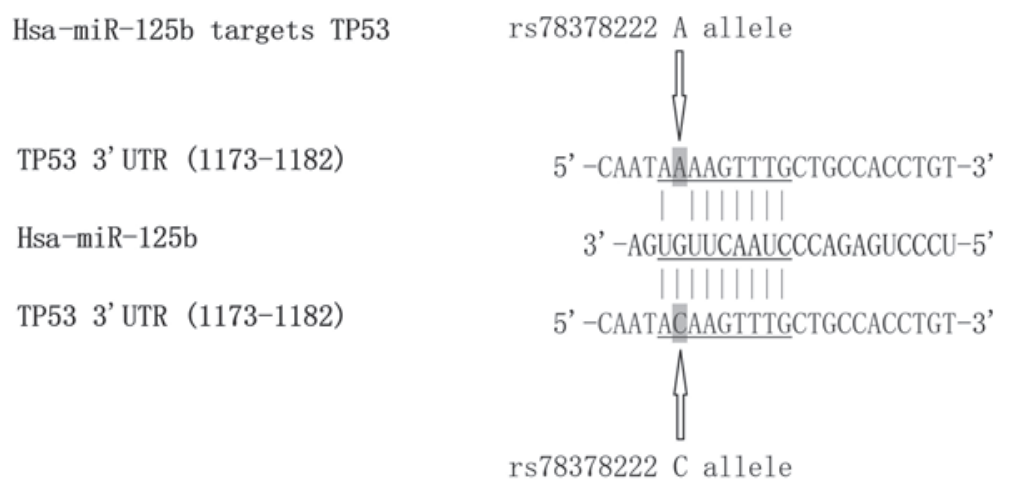

Figure 1. Replacement with C introduces a novel potential binding site in the 3'-UTR of TP53 with consecutive 8-bp perfect match, indicating a 'gain-of-function' role of rs78378222 polymorphism in regulating the expression of TP53. miR, microRNA; TP53, tumor protein p53; 3'-UTR, 3'-untranslated region.

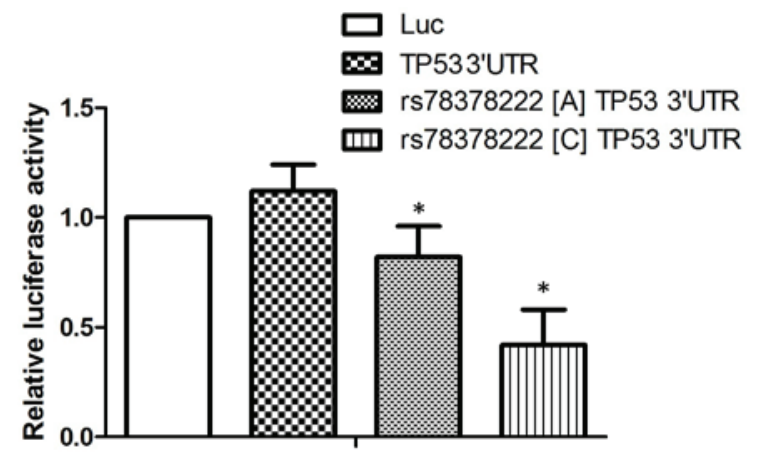

Figure 2. Transfection of wild type TP53 3'-UTR significantly lowered the luciferase activity of the miR-125b overexpressing cells, compared with the scramble controls. The miR-125b overexpressing cells transfected with the construct containing the minor allele of rs78378222 polymorphism was even lower than those transfected with wild type, suggesting that miR-125 may negatively regulate the expression of TP53 by binding the 'seed sequence' in the 3'-UTR of the gene. "P $<0.01$ vs. the control. 3'-UTR, 3'-untranslated region; TP53, tumor protein $\mathrm{p} 53$; miR, microRNA.

$\left(1.5 \times 10^{5}\right.$ cells/well). An apoptosis detection kit (Nanjing KeyGen Biotech. Co. Ltd., Nanjing, China) was used to determine apoptosis using Annexin V/propidium iodide staining. The specimens were cultured at room temperature for $15 \mathrm{~min}$ in darkness and analyzed by flow cytometry (BD Influx ${ }^{\mathrm{TM}}$ Cell Sorter; BD Biosciences, Franklin Lakes, NJ, USA). CellQuest software (BD Biosciences) was used to analyze the results.

Western blot analysis. Protein extraction from SRA01/04 and primary cells was performed by incubation with lysis buffer containing $1 \%$ NP-40, $0.1 \%$ sodium dodecyl sulfate (SDS), $2 \mathrm{mg} / \mathrm{ml}$ aprotinin, $1 \mathrm{mM}$ phenylmethane sulfonyl fluoride and $150 \mathrm{mM} \mathrm{NaCl}$ at $4^{\circ} \mathrm{C}$ for $30 \mathrm{~min}$. A BCA Protein assay kit (Bio-Rad Laboratories, Inc., Hercules, CA, USA) was used to quantify the protein levels. The separation of protein extracts $(20 \mu \mathrm{g})$ was performed via 10\% SDS-polyacrylamide gel electrophoresis (Roche Applied Science, Penzberg, Germany) and transferred onto polyvinylidene difluoride membranes (PerkinElmer, Inc., Waltham, MA, USA). Membranes were blocked in Tris-buffered saline containing $0.05 \%$ Tween 20 (TBST; BioSharp, Hefei, China) with 5\% non-fat milk, $2.7 \mathrm{mmol} / \mathrm{l} \mathrm{KCl}, 137 \mathrm{mmol} / \mathrm{l} \mathrm{NaCl}$ and $25 \mathrm{mmol} / \mathrm{l}$ Tris- $\mathrm{HCl}$ $(\mathrm{pH} 7.5)$ at $37^{\circ} \mathrm{C}$ for $1 \mathrm{~h}$. The membranes were then incubated
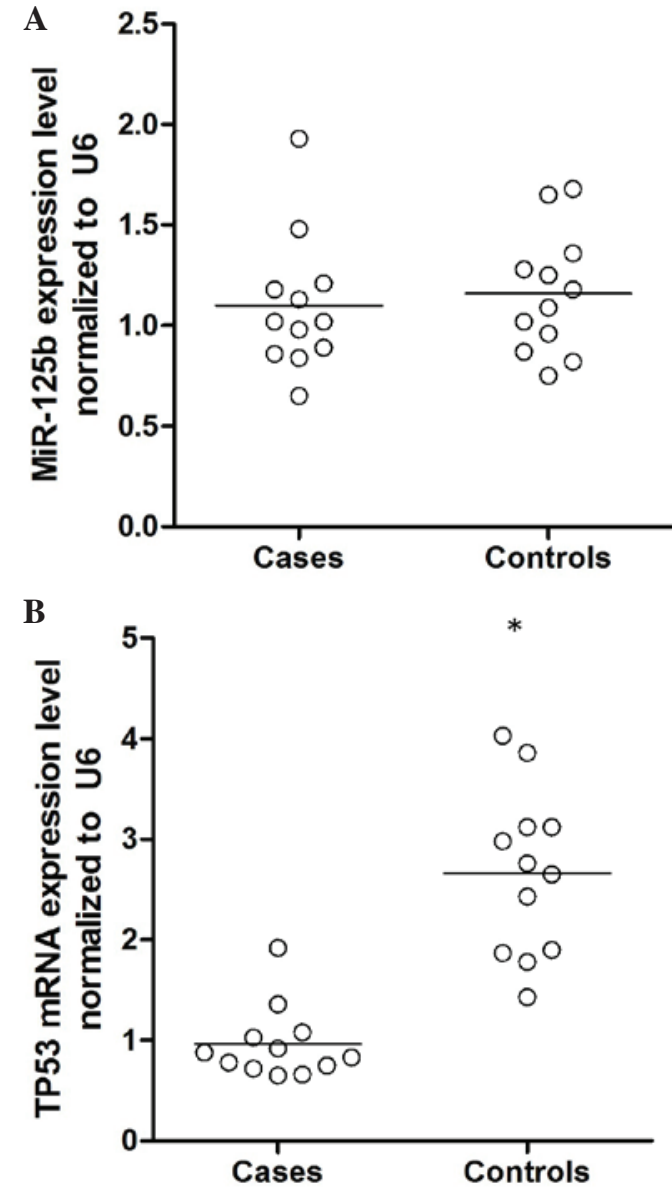

Figure 3. Expression level of miR-125b in cataract and control epithelial cells. (A) miR-125b was comparable between the two groups. (B) The mRNA expression of TP53 was significantly higher in the cataract group compared with the control. "P<0.01 vs. the control. miR, microRNA; TP53, tumor protein $\mathrm{p} 53$.

with primary antibodies, mouse monoclonal against $\beta$-actin (1:8,000; Santa Cruz Biotechnology, Inc., Dallas, TX, USA; cat. no. sc-47778) or rabbit polyclonal against TP53 (1:3,000; Santa Cruz Biotechnology, Inc.; cat. no. 6243) in TBST with $5 \%$ non-fat milk at $4^{\circ} \mathrm{C}$ overnight. Following washes with TBST three times, membranes were cultured with horseradish peroxidase-conjugated goat anti-rabbit polyclonal $(1: 15,000$; Invitrogen; Thermo Fisher Scientific, Inc.; cat. no. Q11402MP) 

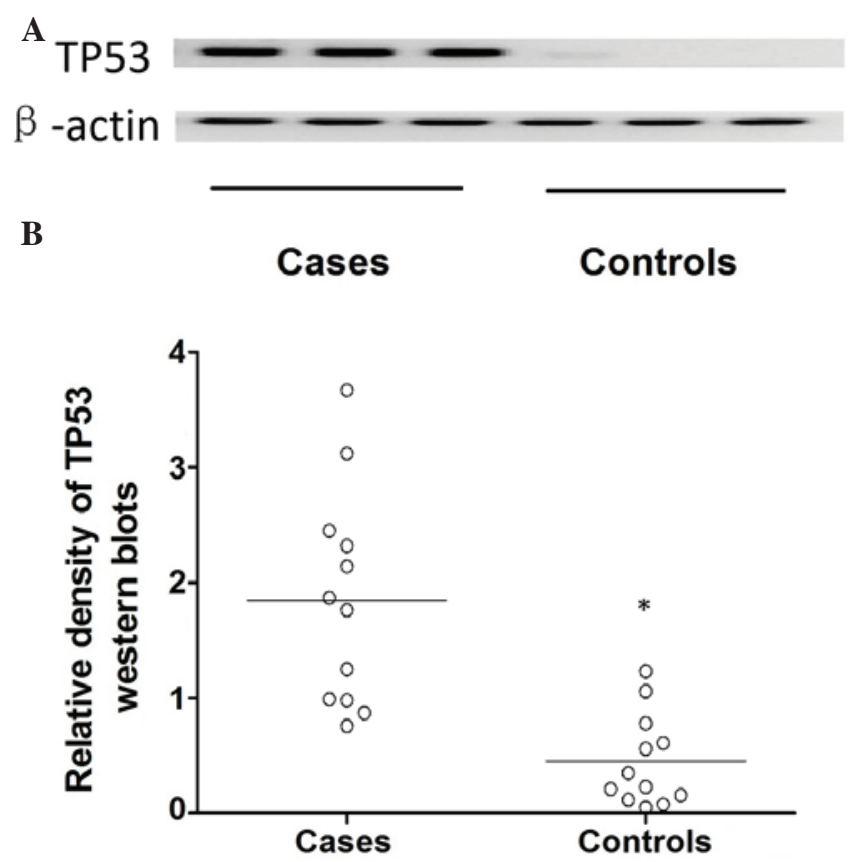

Figure 4. TP53 in cataract epithelial cells. (A) Expression of TP53 protein was determined by using western blot analysis and (B) the target bands on the western blot were quantified by analyzing the relative density, which demonstrated that the protein expression level of TP53 was significantly higher in the cataract group compared with the control group. ${ }^{*} \mathrm{P}<0.01$ vs. the control. TP53, tumor protein $\mathrm{p} 53$.
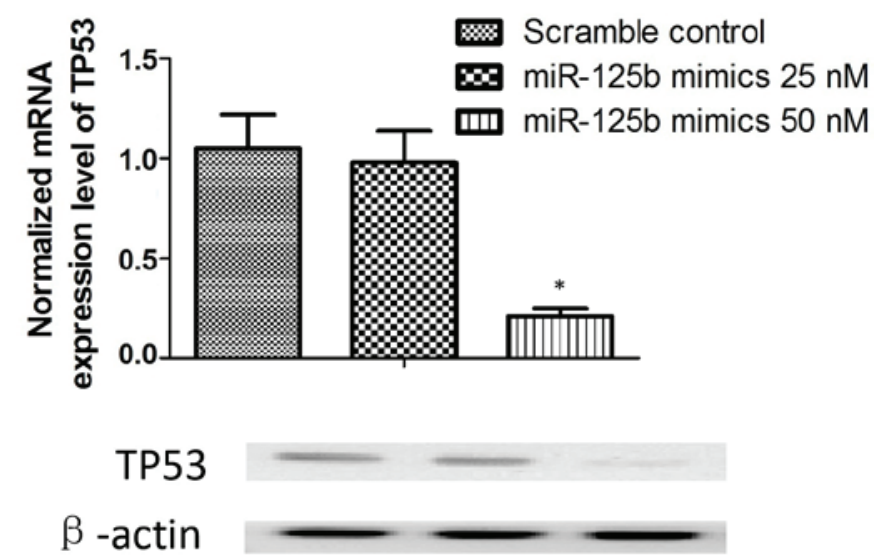

Figure 5. Effect of miR-125b mimics on TP53 expression levels. Transfection of $25 \mathrm{nM}$ mimics did not significantly alter the expression of TP53, $50 \mathrm{nM}$ miR-125b mimics substantially reduced mRNA and protein expression of TP53 in the lens epithelial cells. "P<0.01 vs. the control.miR, microRNA; TP53, tumor protein $\mathrm{p} 53$.

and goat anti-mouse (1:10,000; Santa Cruz Biotechnology, Inc.; cat. no. sc-2005) secondary antibodies for $1 \mathrm{~h}$ at room temperature. Membranes were washed again with TBST, then observed using a SuperSignal West Pico Chemilumunescent Substrate enhanced chemiluminescence kit (Thermo Fisher Scientific, Inc.) and analyzed with ImageJ 1.48 (imagej.nih. gov/ij/).

Statistical analysis. The differences in microarray data were compared between the two groups using the P-values corrected by false discovery rate, which were below 0.05

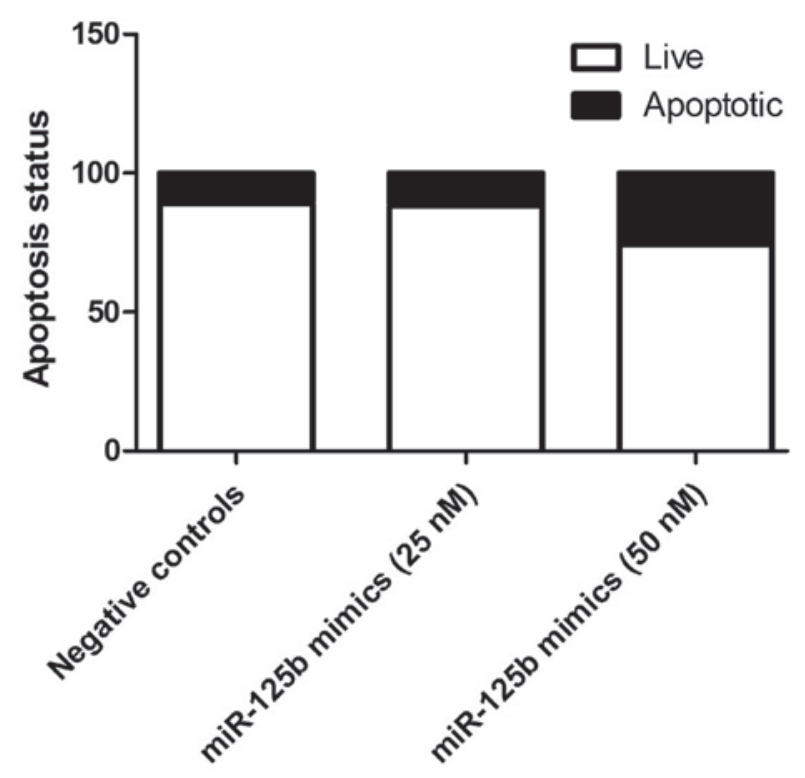

Figure 6. miR-125b significantly induces apoptosis in the epithelial cells, as compared with the control cells. " $\mathrm{P}<0.01$ vs. the control. miR, microRNA.

obtained by significant analysis of microarrays software (statweb.stanford.edu/ tibs/SAM/). SPSS software version 16.0 (SPSS, Inc., Chicago, IL, USA) and an independent samples t-test were used to estimate differences between two groups. $\mathrm{P}<0.05$ was considered to indicate a statistically significant difference.

\section{Results}

Effect of rs78378222 on the binding site in the 3-UTR of TP53. miRNAs negatively regulate the expression of target genes by binding to the 'seed sequence' in the gene 3'-UTR. Variants within or nearby the 'seed sequences' may compromise or enhance miRNA/mRNA interaction leading to either 'loss-of-function' or 'gain-of-function' effects. The present study evaluated the potential effect of the rs78378222 polymorphism in the 3'-UTR of TP53 on miRNA-125b-induced apoptosis of lens epithelial cells, and the association between the rs78378222 polymorphism and the risk of age-associated cataracts. Initially, sequences of mature miR-125b and TP53 (wild-type and polymorphic) were analyzed and compared. As presented in Fig. 1, replacement of A with $\mathrm{C}$ (at position 1177) introduces a novel potential binding site in the 3 '-UTR of TP53 with consecutive 8-bp perfect match, indicating that the rs78378222 polymorphism results in a 'gain-of-function' to regulate TP53 expression.

Effect of rs78378222 on TP53 expression. To examine the effect of the rs78378222 polymorphism on TP53 expression, the full length TP53 3'-UTR was subcloned and inserted into a pcDNA3 vector containing a firefly luciferase gene downstream, and the minor allele of the rs78378222 polymorphism (C) was introduced. The results of the luciferase assay demonstrated that transfection with wild type TP53 3'-UTR (A) significantly reduced the luciferase activity of the miR-125b overexpressing cells, compared with the scramble controls. 
Furthermore, the miR-125b overexpressing cells transfected with the construct containing the minor allele of rs78378222 polymorphism (C) was reduced further than those transfected with wild type 3'-UTR, suggesting miR-125 negatively regulates the expression of TP53 by targeting the 3'-UTR of the gene $(\mathrm{P}<0.01$; Fig. 2$)$, and introduction of a novel binding site caused by the rs78378222 polymorphism further promoted the negative regulatory association between miR-125 and TP53.

$m R N A$ levels of miR-125b and TP53. To further confirm this hypothesis, epithelial cells were collected from patients with age-associated cataracts and controls, and the mRNA expression levels of miR-125b and TP53 were determined. As demonstrated in Fig. 3, the expression level of miR-125b was comparable between the two groups, whereas the mRNA expression level of TP53 was significantly higher in the cataract group compared with the control $(\mathrm{P}<0.01)$. Additionally, the expression of TP53 protein was determined using western blot analysis (Fig. 4). The target bands were quantified by analyzing their relative density, which demonstrated that the protein expression level of TP53 was significantly higher in the cataract cases compared with the control group $(\mathrm{P}<0.01$; Fig. 4).

Regulatory association between miR-125b and TP53. To further investigate the regulatory association between miR-125b and TP53, miR-125b mimics were transfected into cultured SRA01/04 epithelial cells. Transfection with $25 \mathrm{nM}$ mimics was not identified to significantly alter the expression of TP53; however, $50 \mathrm{nM}$ miR-125b mimics substantially reduced the mRNA and protein expression levels of TP53 in the lens epithelial cells $(\mathrm{P}<0.01$; Fig. 5). Additionally, compared with negative controls, miR-125b (50 nM) significantly induced apoptosis in the epithelial cells (Fig. 6).

\section{Discussion}

Previous investigation demonstrated that miRNAs are an important group of gene regulators. The complete miRNA transcriptome in human lens cells is unclear, although miRNAs have been detected in a variety of mammalian organs and tissues, including the eyes. To the best of our knowledge, the present study is the first to investigate miRNAs in the central epithelium of cataractous and transparent human lens. Determining the mutations that lead to the development of age-associated cataracts may improve understanding of the mechanisms that mediate cataractogenesis, and provide novel insights into the development and physiology of the normal lens. Furthermore, functional analysis of candidate variants is a critical measure to understand the molecular defects involved in age-associated cataracts, which has a strong genetic component to its etiology. This may aid in elucidating novel therapeutic strategies to delay lens opacification. The present study evaluated the potential effect of TP53 3'-UTR rs78378222 on the miR-125b-induced apoptosis of lens epithelial cells, and the association between the rs78378222 polymorphism and the risk of the development of age-associated cataracts. Initially, the sequences of mature miR-125b and TP53 (wild-type and polymorphic) were analyzed and compared. Replacement of A with $\mathrm{C}$ introduces a novel potential binding site in the 3'-UTR of TP53 with a consecutive 8 -bp perfect match, indicating that the rs78378222 polymorphism creates a 'gain-of-function' effect to regulate TP53 expression via miR-125b.

As a highly conserved miRNA expressed throughout an array of species ranging from nematode to human, miR-125b acts as either a repressor or promoter of gene expression, and is associated with various diseases (24). By acting on a range of different transcription factors (25), growth factors (26) and matrix metalloproteinases $(27,28)$, miR-125b is important for various cellular processes, including cell proliferation, differentiation and apoptosis. The current study identified TP53 as a target of miR-125b, and replacement with the rs78378222 polymorphism minor allele (C) significantly promoted interaction between the miR-125b and TP53 mRNA, as demonstrated by a luciferase assay. Furthermore, the present study demonstrated that transfection of lens epithelial cells with $25 \mathrm{nM}$ mimics did not significantly alter the expression of TP53, whereas $50 \mathrm{nM}$ miR-125b mimics significantly reduced the mRNA and protein expression level of TP53 in the lens epithelial cells compared with negative control miRNA.

TP53 is a well-studied pro-apoptotic protein in vivo. Prior to 2011, no genome-wide association study had demonstrated a significant correlation between any cancer and a TP53 polymorphism germline (other than variants of Li-Fraumeni syndrome) (29). The rs78378222 TP53 polymorphism was previously demonstrated to be significantly associated with breast cancer (30). An independent study confirmed that rs78378222 was correlated with a 3.5-fold higher risk of glioma development (31). Stacey et al (32) demonstrated that the transcript levels of TP53 expressed by rs78378222 [A/C] heterozygotes was lower than that in wild type homozygotes in human blood samples. rs78378222 is located in the fifth nucleotide of the TP53 polyadenylation signal and was initially hypothesized to be required for a wide range of processes, including polyadenylation machinery recognition, cleavage, polyadenylation and transport of mature mRNAs to the cytoplasm. The current study demonstrated that replacement with $\mathrm{C}$ in the sequence introduces a novel potential miRNA binding site in the TP53 3'-UTR, with a consecutive 8-bp perfect match, indicating that rs78378222 creates a 'gain-of-function' effect. miR-125 is another important regulator of TP53, and the minor allele (C) of the rs78378222 polymorphism 'generates' a novel perfect match binding site for miR-125b in the 3 '-UTR of TP53. Additionally, the present study demonstrated that the expression level of miR-125b was comparable in epithelial cells from patients with cataracts and controls, whereas the mRNA expression level of TP53 was significantly higher in the cataract group compared with the controls, suggesting that the polymorphism significantly promotes interaction between miR-125b and TP53.

In conclusion, the current study demonstrated that the minor allele (C) of the rs78378222 polymorphism introduces a potential novel miR-125b binding site in the 3'-UTR of TP53, with a consecutive 8 -bp perfect match, indicating that the rs78378222 polymorphism induces a 'gain-of-function' effect to regulate of TP53 expression via miR-125b. Furthermore, the present study demonstrated that miR-125b is a novel negative regulator of TP53 in vivo, which may be a mechanism 
of age-associated cataract development. miR-125b may be a potential therapeutic target for the management of age-associated cataracts. Further research in transgenic animals may be able to increase understanding of the importance of miR-125b and confirm the results of the present study.

\section{Acknowledgements}

The present study was supported by the National Natural Science Foundation of Youth Science Foundation (grant no. 51302176).

\section{References}

1. Pascolini D, Mariotti SP, Pokharel GP, Pararajasegaram R, Etya'ale D, Négrel AD and Resnikoff S: 2002 global update of available data on visual impairment: A compilation of population-based prevalence studies. Ophthalmic Epidemiol 11: $67-115,2004$

2. Congdon N, O'Colmain B, Klaver CC, Klein R, Muñoz B, Friedman DS, Kempen J, Taylor HR and Mitchell P; Eye Diseases Prevalence Research Group: Causes and prevalence of visual impairment among adults in the United States. Arch Ophthalmol 122: 477-485, 2004.

3. West S: Epidemiology of cataract: Accomplishments over 25 years and future directions. Ophthalmic Epidemiol 14: 173-178, 2007.

4. Resnikoff S, Pascolini D, Etya'ale D, Kocur I, Pararajasegaram R, Pokharel GP and Mariotti SP: Global data on visual impairment in the year 2002. Bull World Health Organ 82: 844-851, 2004.

5. Cruciani F, Amore F, Albanese G and Anzidei R: Investigation about causes of blindness and low vision among members of Blind and Visually Impaired Italian Union (UICI). Clin Ter 162: e35-e42, 2011.

6. WHO releases the new global estimates on visual impairment Accessed on, http://www.who.int/blindness/en/2011.

7. Chen K and Rajewsky N: The evolution of gene regulation by transcription factors and microRNAs. Nat Rev Genet 8: 93-103, 2007.

8. Peters L and Meister G: Argonaute proteins: Mediators of RNA silencing. Mol Cell 26: 611-623, 2007.

9. Karali M, Peluso I, Marigo V and Banfi S: Identification and characterization of microRNAs expressed in the mouse eye. Invest Ophthalmol Vis Sci 48: 509-515, 2007.

10. Ryan DG, Oliveira-Fernandes M and Lavker RM: MicroRNAs of the mammalian eye display distinct and overlapping tissue specificity. Mol Vis 12: 1175-1184, 2006.

11. Maegdefessel L: The emerging role of microRNAs in cardiovascular disease. J Intern Med 276: 633-644, 2014.

12. Lee YH, Kim SY and Bae YS: Upregulation of miR-760 and miR-186 is associated with replicative senescence in human lung fibroblast cells. Mol Cells 37: 620-627, 2014.

13. Mimura S, Iwama $\mathrm{H}$, Kato $\mathrm{K}$, Nomura $\mathrm{K}$, Kobayashi $\mathrm{M}$, Yoneyama H, Miyoshi H, Tani J, Morishita A, Himoto T, et al: Profile of microRNAs associated with aging in rat liver. Int J Mol Med 34: 1065-1072, 2014.

14. Khee SG, Yusof YA and Makpol S: Expression of senescence-associated microRNAs and target genes in cellular aging and modulation by tocotrienol-rich fraction. Oxid $\mathrm{Med}$ Cell Longev 2014: 725929, 2014

15. Hughes AE, Bradley DT, Campbell M, Lechner J, Dash DP, Simpson DA and Willoughby CE: Mutation altering the miR-184 seed region causes familial keratoconus with cataract. Am J Hum Genet 89: 628-633, 2011
16. Kota J, Chivukula RR, O'Donnell KA, Wentzel EA, Montgomery CL, Hwang HW, Chang TC, Vivekanandan P, Torbenson M, Clark KR, et al: Therapeutic microRNA delivery suppresses tumorigenesis in a murine liver cancer model. Cell 137: 1005-1017, 2009.

17. Lanford RE, Hildebrandt-Eriksen ES, Petri A, Persson R, Lindow M, Munk ME, Kauppinen S and Ørum H: Therapeutic silencing of microRNA-122 in primates with chronic hepatitis C virus infection. Science 327: 198-201, 2010.

18. Wang Z, Rajaraman P, Melin BS, Chung CC, Zhang W, McKean-Cowdin R, Michaud D, Yeager M, Ahlbom A, Albanes D, et al: Further confirmation of germline glioma risk variant rs78378222 in TP53 and its implication in tumor tissues via integrative analysis of TCGA data. Hum Mutat 36: 684-688.

19. Guan X, Wang LE, Liu Z, Sturgis EM and Wei Q: Association between a rate novel TP53 variant (rs78378222) and melanoma, squamous cell carcinoma of the head and neck and lung cancer susceptibility in non-Hispanic Whites. J Cell Mol Med 17: 873-878, 2013

20. Long AC, Bomser JA, Grzybowski DM and Chandler HL: All-trans retinoic acid regulates cx43 expression, gap junction communication and differentiation in primary lens epithelial cells. Curr Eye Res 35: 670-679, 2010.

21. Carlson RV, Boyd KM and Webb DJ: The revision of the Declaration of Helsinki: Past, present and future. Br J Clin Pharmacol 57: 695-713, 2004.

22. Chylack LT Jr, Wolfe JK, Singer DM, Leske MC, Bullimore MA, Bailey IL, Friend J, McCarthy D and Wu SY: The lens opacities classification system III. The longitudinal study of cataract study group. Arch Ophthalmol 111: 831-836, 1993.

23. Ferracin M, Bassi C, Pedriali M,Pagotto S, D'Abundo L, Zagatti B, Corrà $\mathrm{F}$, Musa G, Callegari E, Lupini L, et al: miR-125b targets erythropoietin and its receptor and their expression correlates with metastatic potential and ERBB2/HER2 expression. Mol Cancer 12: 130, 2013.

24. Sun YM, Lin KY and Chen YQ: Diverse functions of miR-125 family in different cell contexts. J Hematol Oncol 6: 6, 2013.

25. Bousquet M, Nguyen D, Chen C, Shields L and Lodish HF: MicroRNA-125b transforms myeloid cell lines by repressing multiple mRNA. Haematologica 97: 1713-1721, 2012.

26. Ge Y, Sun Y and Chen J: IGF-II is regulated by microRNA-125b in skeletal myogenesis. J Cell Biol 192: 69-81, 2011.

27. Bi Q, Tang S, Xia L, Du R, Fan R, Gao L, Jin J, Liang S, Chen Z, $\mathrm{Xu} \mathrm{G}$, et al: Ectopic expression of MiR-125a inhibits the proliferation and metastasis of hepatocellular carcinoma by targeting MMP11 and VEGF. PLoS One 7: e40169, 2012.

28. Xu N, Zhang L, Meisgen F, Harada M, Heilborn J, Homey B, Grandér D, Ståhle M, Sonkoly E and Pivarcsi A: MicroRNA-125b down-regulates matrix metallopeptidase 13 and inhibits cutaneous squamous cell carcinoma cell proliferation, migration, and invasion. J Biol Chem 287: 29899-29908, 2012.

29. Thomas DC, Haile RW and Duggan D: Recent developments in genomewide association scans: A workshop summary and review. Am J Hum Genet 77: 337-345, 2005.

30. Rao AK, Vinothkumar V, Revathidevi S, Arunkumar G, Manikandan M, Arun K, Rajkumar KS, Ramani R, Ramamurthy R and Munirajan AK: Abscence of the TP53 poly-A signal sequence variant rs78378222 in oral, cervical and breast cancers in South India. Asian Pac J Cancer Prev 15: 9555-9556, 2014.

31. Egan KM, Nabors LB, Olson JJ, Monteiro AN, Browning JE, Madden MH and Thompson RC: Rare TP53 genetic variant associated with glioma risk and outcome. J Med Genet 49: 420-421, 2012.

32. Stacey SN, Sulem P, Jonasdottir A, Masson G, Gudmundsson J, Gudbjartsson DF, Magnusson OT, Gudjonsson SA, Sigurgeirsson B, Thorisdottir $\mathrm{K}$, et al: A germline variant in the TP53 polyadenylation signal confers cancer susceptibility. Nat Genet 43: 1098-1103, 2011. 